\title{
Implications of single field inflation in general cosmological scenarios on the nature of dark energy given the swampland conjectures
}

\author{
Oem Trived: $*$ \\ School of Arts and Sciences, Ahmedabad University,Ahmedabad 380009, India
}

(Dated: January 21, 2021)

\begin{abstract}
Swampland Conjectures have attracted quite some interest in the Cosmological Community. They have been shown to have wide ranging implications, like Constraints on Inflationary Models, Primordial Black Holes etc. to name a few. A particularly revealing insight on dark energy also shows that one can have the dark energy equation of state for a quintessence scenario to be signficantly different than -1 after one takes into account the refined dS conjecture. Another interesting issue with the swampland conjectures is that they have been shown to be incompatible with single field inflationary models in GR based cosmology. In our previous work we have, however, showed that single field inflationary models are quite compatible with swampland conjectures in their usual string theoretic form in a large class of modified cosmological scenarios. Building on that work, we now show that in modified cosmological scenarios where the early universe expansion was driven by single field inflation, one can have the dark energy equation of state to be significantly different from -1 even if we just take into account the original dS conjecture, let alone the refined form of that. We thereby show that one does not need to apply a step function approach towards inflation in order to have an observable distinction between constant and non constant dark energy models in the context of the swampland conjectures.
\end{abstract}

\section{INTRODUCTION}

Inflationary Cosmology has achieved a tremendous amount of success in describing the Very Early Universe [1 5]. .A huge amount of inflationary models [6 - 15] have been shown to be very consistent with the most recent datasets on the early universe [16 20]. The consistency of Inflation with observational data for large amount of diversely motivated models is a really eye catching property of Inflationary Cosmology. Besides explaining various problems of the early universe in standard big bang cosmology, inflation has also provided a way to understand the current dark energy epoch of the universe. The idea of Quintessential Inflation has garnered quite a lot of interest recently and in this, the Inflaton field itself becomes the cause of the accelerated expansion of the universe today [21 29]. The idea of Quintessence, like Inflation, has been thoroughly studied in Modified Cosmologies as well 30 35].

In recent years, a quest for a unified theory of everything has garnered a lot of attention in high energy physics. Probably the most well known of these "theory of everything" candidates, is String Theory [36 44]. It is one of the most viable approaches for a theory of everything for many (if not the most viable), hence one would expect that String theory has some implications for Cosmology and could also hence allow us to understand both the very early universe and it's subsequent evolution in more detail. So it's no surprise that a substantial amount of work has been done on studying the cosmological implications of String theory [45 52]. One curious feature of this theory is the extremely high amount of

*om.t@ahduni.edu.in vacua states that is posits, going around $\mathcal{O}\left(10^{500}\right)$ and this constitutes the so called "String Landscape". But then, a fundamental question that arises is how can one distinguish between low energy effective field theories which are consistent with String theory and those which are not. In a bid to understand this question in more detail, Vafa in [53] gave the term "Swampland" to refer to the set of all low energy EFT's which are inconsistent with the String paradigm. As String theory is (as a consequence of being a theory of everything) seen as a viable quantum gravitational setup by string theorists, then this would go on to mean that the theories in the swampland are also not viable with a self consistent theory of Quantum gravity. Further, to know as to if a given low energy EFT belongs in the swampland, a number of field theoretic criterion known as the "Swampland conjectures" have been proposed in recent years 54 58. The prominent Swampland Conejctures which gathered immediate interest in the context of Cosmology were :

1 : Swampland Distance Conjecture (SDC) : This conjecture limits the field space of validity of any effective field theory [55]. This sets a maximum range traversable by the scalar fields in an EFT as

$$
\Delta \phi \leq d \sim \mathcal{O}(1)
$$

where we are working in the Planck Units $m_{p}=1$ where $m_{p}$ is the reduced Planck's constant, $\mathrm{d}$ is some constant of $\mathcal{O}(1)$, and $\phi$ is the Scalar Field of the EFT.

2 Swampland De Sitter Conjecture (SDSC) : This Conjecture states that it is not possible to create dS Vacua in String Theory [54]. The conjecture is a result of the observation that it has been very hard to generate dS Vacua in String Theory [59, 60] ( While it has been 
shown that creating dS Vacua in String Theory is possible in some schemes, like the KKLT Construction [61]). The Conjecture sets a lower bound on the gradient of Scalar Potentials in an EFT,

$$
\frac{\left|V^{\prime}\right|}{V} \geq c \sim \mathcal{O}(1)
$$

where $\mathrm{c}$ is some constant of $\mathcal{O}(1)$, and $\mathrm{V}$ is the scalar Field Potential. Another " refined" form of the Swampland De Sitter Conjecutre (RSDSC) places constraints on the Hessian of the Scalar Potential (a finding which first appeared in [58] and later in [62]). Expressed in $m_{p}=1$ units, it reads

$$
\frac{V^{\prime \prime}}{V} \leq-c^{\prime} \sim \mathcal{O}(1)
$$

where $c^{\prime}$ is again some constant of Order 1 .

The conjecture has had quite fascinating implications for single field inflation, particularly those regimes in a General Relativistic Cosmology. It was shown in 63 that the swampland conjectures Eq. (1-3) are not consistent with the cosmological data on single field inflation in a GR based cosmology and for them to be viable with this form of Inflation, the string theorymotivated definitions of the conjectures would have to change. A lot of work has since then been done to understand the issues of the distance and de sitter conjectures with single field Inflation [64 68. Besides these conjectures, another recently proposed swampland conjecture by the name of the "Trans Planckian Censorship Conjecture(TCC)" [57], implies that single field Inflation in a GR Based Cosmology would have to be severely fine tuned in order for it to not lie in the swampland [69]. If Inflation is plagued with severe fine tuning problems itself, which were the kind of issues in standard big bang cosmology which prompted work on Inflation in the fist case, then it is certainly a very dire situation for Inflationary Cosmology keeping in mind these conjectures. A lot of work has been done in order to understand the issues of single field GR based inflation and the TCC in more detail 70 79. A particularly interesting observation was made in [80], which showed that the TCC can be derived from the Distance conjecture (1) considering that criterion to be true. The swampland conjectures have also had some pretty interesting implications on the paradigm of eternal inflation [81, 86].

The revelations from these works seemingly show that Inflation and the Swampland conjectures are in unavoidable loggerheads with each other. However, the true scenario is that it is specifically Single Field Supercooled Inflation in GR Based Cosmology, which has serious issues with the swampland criterion. Indeed, it has been shown that Inflation, even for single field cases, can be quite consistent with the swampland conjectures in other regimes of Inflation. One can take the example of warm inflation [8], which is on amicable terms with these conjectures even for single field cases in a GR based cosmology 87 94]. Single field inflation in other cosmological scenarios which are motivated by vastly different reasons, like string theory motivated Braneworld Models 95 97] or regimes like Gauss-Bonet Cosmology [98] which are motivated by geometric requirements, have been aptly studied in the context of the swampland conjectures and have proved to be on good terms with these criterion as well [99 104]. Particularly in 64], it was shown that single field (cold) Inflation in a large class of non-GR based cosmologies can be quite easily consistent with the swampland conjectures. For example, this work also tangentially showed that single field inflation in loop quantum cosmology 9, 105, 106 and Chern-Simons Cosmology [107] can also be on good terms with the swampland conjectures. Finally, it has also been shown that perhaps the Inflationary scenarios which fits the swampland conjectures the best are multi field models [108, 109].

The implications of the swampland conjectures on the current dark energy epoch of the universe are quite interesting as well. Particularly the dS conjecture (2) is in stark opposition of a cosmological constant form of dark energy and favours quintessence to be a better realization of dark energy 110 120. It was however shown in [121], that all the existing string theory based models of quintessence with an exponential potential or combinations of exponential potentials are ruled out by current observations, implying that the swampland de Sitter conjecture is in tension with viable quintessence models, similarly to the single-field slow-roll inflation in a GR based cosmological scenario. The findings of that paper were then further confirmed in [122] with even more data. Furthermore, a significant amount of interest has also gone towards investigating non conventional quintessence models in the view of the swampland conjectures, with both multi-field and modified cosmological models having been studied in this regard 111, 123, 124]. There has also been some very insightful work on studying quintessence based dark energy models in the view of the Hubble Tension [125-131] as a litmus test for the swampland conjectures in [132, 133]. Hence, there are quite a few reasons to consider quintessential inflation as a viable paradigm of dark energy and more so in the context of the swampland conjectures. If it is indeed the case for the current epoch of the universe, one might expect that the equation of state parameter of the universe can be quite different from the usual -1 in the case of a dS universe(and so a cosmological constant form of dark energy). It has, however, been shown to not quite be the case. Considering the universe went through (cold) single filed inflation in it's very early epoch and taking into account the swampland conjectures (1-3), it was shown in 134] that equation of state parameter can only diverge quite negligibly from -1 . The point to note here is that this work focused on a GR based cosmology 
and the work done on single field inflation based in a GR based cosmology 63. We have however showed in a recent paper 64 that swampland conjectures have pretty different implications on single field inflation in a general class of modified cosmologies. Hence, in this paper we will be building on that work and discuss the implications that the Distance and the dS conjectures have on quintessence models in modified cosmological scenarios, given that the early universe expansion was driven by (cold) single field inflation. In the next section, we will be shortly reviewing the important aspects of the work discussed in [64]. In Section III, we will discuss the implications of that on the equation of state parameter of the universe and see how one can get a viable distinction between constant and non-constant dark energy models through the equation of state parameter in the view of the swampland conjectures, without even having to rely to a step-function approach to inflation. We will then conclude this work in section IV with some general remarks.

\section{SINGLE FIELD INFLATION IN GENERAL COSMOLOGIES AND THE SWAMPLAND CONJECTURES}

The central points of conflict which have been raised between single field Inflation and the primary swampland conjectures were explored deeply in [63]. The most serious issues which arose between these two paradigms were based on the string theory-motivated definitions of the swampland conjectures, being in grave conflict with the available observational data on single field inflation. We will quickly go over these issues now. One of the central quantities which one encounters while studying single field inflation are the slow roll parameters. A particularly important slow roll parameter is the $\epsilon$ parameter, which can be written in $m_{p}=1$ units in a GR based cosmology as,

$$
\epsilon=\frac{1}{2}\left(\frac{V^{\prime}}{V}\right)^{2}
$$

During inflation one needs $\epsilon<<1$ [2], and hence, it's immediately evident that the smallness requirement of the $\epsilon$ parameter is in direct conflict with the dS conjecture (2). Another issue concerns the e-fold number during Inflation, which is given the GR paradigm roughly as

$$
N \approx \frac{V \Delta \phi}{V^{\prime}}
$$

The e-fold number is a good measure of telling how much Inflation occurs in some certain model. For inflation to solve the problems of the standard big bang cosmology, one needs at least around 50 to 60 e-folds of inflationary expansion [17]. But it can again be immediately clear, that if one seriously considers the distance and dS conjectures (1-2), then the e-fold number would not even be greater than unity. This is an incredible assertion and one which would raise questions over the existence of any kind of single field inflationary expansion in the context of the swampland conjectures. Further in [63], the following relation between the scalar spectral index [16, 17] and the $c$ and $c^{\prime}$ parameters from the swampland conjectures (1-2), was derived

$$
1-n_{s}=\left[3 c^{2} ; 2 c^{\prime}\right]
$$

Using the data on Inflation [17], one can hence deduce that $c \leq \mathcal{O}(0.1)$ and $c^{\prime} \leq \mathcal{O}(0.01)$, which is clearly very different then the $\mathcal{O}(1)$ estimates of these parameters in their string theoretic form (2-3). The above equation (6), had it's roots in the following relations between the slow roll and swampland conjecture parameters,

$$
\epsilon \geq \frac{c^{2}}{2}
$$

$$
\eta \leq-c^{\prime}
$$

This clearly shows that the swampland conjectures in their string theoretic form are not consistent with the data on single field inflation, in a general relativistic cosmology. While there have been works which have dealt with the swampland conjectures in some particular cosmologies [99, 101, 102, 104], in 64] it was shown using a very general approach that the swampland conjectures will not have any unavoidable conflicts with single field inflation in a large class of non GR cosmological scenarios. The general Friedmann equation we considered in [64], is given by

$$
F(H)=\frac{\rho}{3}
$$

where we are working in $m_{p}=1$ units. Here $\mathrm{F}(\mathrm{H})$ is some function of the Hubble Parameter and $\rho$ is the energy density of the universe. Friedmann equations of this form have been used to study Inflation in detail in the paradigm of the Exact Solution Approach [135, 136], which is a generalization of the Hamilton-Jacobi Approach to Inflation to general cosmologies 137. Using this method, we can revaluate whether the issues which persist between single field inflation and the swampland conjectures in a GR based cosmology still hold tight in large class of cosmologies described by the general friedmann equation (9) (for more details on how general our friedmann equation in consideration is, please refer to [135, 136]). The work in 64 showed that the main issues of conflict between single field inflation and swampland conjectures highlighted in [63] , only necessarily hold good in a GR based cosmology and are not sources of unavoidable loggerheads between these two paradigms in the more general cosmologies described by the Friedmann equation above.

The $\epsilon$ parameter, for example, can be as small as is 
needed for a period of inflationary expansion as in general the $\epsilon$ parameter can be written as [64],

$$
\epsilon=\frac{F H^{\prime} k}{H^{3}}
$$

where $k=\frac{V^{\prime}}{V}$ and considering the dS conjecture (2), $k \geq c \sim \mathcal{O}(1)$. During inflation, one needs $\epsilon<<1$ and we can immediately start to see that in general cosmological scenarios $\left(F(H) \neq H^{2}\right)$, one can easily have the $\epsilon$ parameter as small as needed whilst still satisfying the $\mathrm{dS}$ conjecture requirement $k>\mathcal{O}(1)$. There is no severe issue of an insufficient e-fold number as the e-fold number in this paradigm is given by ,

$$
N \approx \frac{H^{2}}{F} \frac{\Delta \phi}{k}
$$

where again for non-GR cosmologies, the e-fold number can satisfy both the distance and dS conjectures (1-2), and still be high enough for inflation to solve the fine tuning problems of standard big bang cosmology. And finally, the scalar spectral index is related to the $\mathrm{c}$ and $c^{\prime}$ parameters as,

$$
\begin{gathered}
\left(\frac{\left(1-n_{s}\right) H^{2}}{2 F}\right)\left[\frac{1}{\left(3-H \frac{F_{, H H}}{F_{, H}}\right) \frac{H^{\prime}}{H}-\frac{H^{\prime \prime}}{H^{\prime}}}\right]=\frac{F_{, H} H^{\prime}}{F} \geq c \\
\frac{1}{F_{, H}}\left[F_{, H H} H^{\prime}+\eta \frac{F_{, H} H^{2}}{H^{\prime}}\right] \leq-c^{\prime}
\end{gathered}
$$

Again, the above equations (12-13) show that the $n_{s}$ can have it's appropriate observational value [16, 17] whilst still considering the string theoretic $\mathcal{O}(1)$ estimates of the $\mathrm{c}$ and $c^{\prime}$ parameters. For the full discussion on how single field inflation and swampland conjectures can be in a peaceful harmony in general cosmological scenarios in the scope of our considered Friedmann equation (9), please refer to 64 .

\section{SWAMPLAND CONJECTURE IMPLICATIONS ON QUINTESSENCE DARK ENERGY EQUATION OF STATE IN GENERAL COSMOLOGICAL SCENARIOS}

As mentioned earlier, there has been quite a lot of work on Quintessence models in the light of the swampland criterion. The swampland conjectures support the idea that the current dark energy epoch of our universe is due to rolling quintessence field rather than a positive cosmological constant [110]. It was also recently shown that considering the swampland conjectures to be true, one would not observe the equation of state parameter of the universe to be greatly different than it's dS value of -1 [134]. This revelation was made considering a GR based cosmology and as we are talking about only a single quintessence field, this result was also made on the consideration that the early universe expansion occured through single field inflation.

We will now,however, like to show that the equation of state parameter in general cosmological scenarios described by (9), can indeed have a signficantly different value from it's dS scenario even after considering the swampland conjectures. By definition, the equation of state parameter is given by

$$
w=\frac{p}{\rho}
$$

where $\mathrm{p}$ and $\rho$ are the pressure and energy densities of the quintessence field, respectively, given by $p=\frac{\dot{\phi}^{2}}{2}-V(\phi)$ and $\rho=\frac{\dot{\phi}^{2}}{2}+V(\phi)$. Hence,

$$
1+w=\frac{2 \dot{\phi}^{2}}{\dot{\phi}^{2}+2 V}
$$

For a rolling quintessence field, we have [23]

$$
3 H \dot{\phi} \approx-V^{\prime}(\phi)
$$

which allows us to express (15) as

$$
1+w=\frac{2 V^{\prime 2}}{V^{\prime 2}+18 H^{2} V}
$$

Further, for a rolling field in a general cosmological scenario our Friedmann equation (9) becomes

$$
F \approx \frac{V}{3}
$$

and this allows us to further rewrite (17) as

$$
1+w=\frac{2 V^{\prime 2} F}{F V^{\prime 2}+6 V^{2} H^{2}}
$$

Finally, considering the dS conjecture (2) we can write the above equation as

$$
1+w \geq \frac{2 c^{2} F}{F c^{2}+6 H^{2}}
$$

One can now see in the above inequality that for general non-GR based cosmologies $\left(F \neq H^{2}\right)$, w can have quite a different value than it's usual dS case even when we consider the dS conjecture. As one considers general cosmological scenarios, the parameter c can retain it's $\mathcal{O}(1)$ estimate and still be consistent with data on single field inflation. This makes a pivotal difference, as similar analysis for the quintessence field in a GR based cosmology (with $c \approx 0.02[63]$ ) resulted in a quite negligible deviation from -1 for $\mathrm{w}$ [134]. However, the $\mathrm{c}$ parameter retaining it's string theoretic value coupled 
with the fact that one can work with a range of different $\mathrm{F}$ functions corresponding to different cosmologies (again, we recommend [135, 136] for an overview of the generality of the Friedmann equation in consideration), which would then give one a range of model-specific free parameters which could then be tuned to get quite a significant deviation from the dS value of w. It's important to also note that in the Hamilton-Jacobi approach one starts with an ansatz for the Hubble Parameter 137] (contrary to the usual approach to inflationary studies where one starts off with a potential and gets the physically interesting results from that) , and in the generalizations of the Hamilton-Jacobi to general cosmologies [135, 136] one also takes some predefined $\mathrm{F}$ to ascertain the cosmology they want to study inflation in. Hence, the free parameters for the model specific cases generate from these ansatz that one starts off with before making concrete calculations. This shows that in general cosmological scenarios one can have an appropriately observable distinction between constant and non constant dark energy models, whilst still considering the swampland conjectures to hold true in their string theoretic forms. This results in an exciting prospect for experiments which are aiming to measure w to within a percent's accuracy, like DES [138], DESI [139], PFS [140], Euclid [141], HSS 142], LSST [143] and WFIRST [144] .

In [134], a step function approach towards inflation was taken and both the forms of the $\mathrm{dS}$ conjectures were applied in different stages of the inflationary regime owing to the tensions of the dS conjectures individually with single field inflation and the consequent negligible deviation that the $\mathrm{w}$ parameter has for a quintessence scenario when these conjectures are considered in a GR based cosmology. As the dS conjectures and single field inflation do not have any unavoidable conflicts with each other in general cosmological scenarios [64], one does not need adopt the step function approach to inflation in these cosmological scenarios and can have quite a different value of the $\mathrm{w}$ parameter from it's positive cosmological constant case.

\section{CONCLUDING REMARKS AND DISCUSSION}

In this paper we have discussed the status of dark energy quintessence models in general cosmological scenarios whilst considering the swampland conjectures. We started by discussing the core issues of conflict between single fied inflation and swampland conjectues in a GR based cosmology and what these issues have implied for quintessence models. Then we briefly discussed how there are no such unavoidable conflicts between single field inflation and swampland conjectures in general cosmological scenarios, which are in the scope of a particular friedmann equation (9) and where the inflaton (and consequently the quintessence field ) follows a usual KleinGordon form. We then built upon that, and showed that the equation of state parameter in general cosmological scenarios can be significantly different from it's cosmological constant value of -1 and hence, one can make a clear distinction between constant and non constant dark energy models in such cosmologies even after considering the swampland conjectures. We then concluded by noting that the absence of any serious tension between single field inflation and the conjectures in general cosmological scenarios, we hence showed that one does not need to apply any step function approach to single field inflation in order to have an equation of state parameter significantly different from -1 in the context of the swampland conjectures.
[1] Katsuhiko Sato. First-order phase transition of a vacuum and the expansion of the universe. Monthly Notices of the Royal Astronomical Society, 195(3):467-479, 1981.

[2] Daniel Baumann. Tasi lectures on inflation. arXiv preprint arXiv:0907.5424, 2009.

[3] Alan H Guth. Inflationary universe: A possible solution to the horizon and flatness problems. Physical Review D, 23(2):347, 1981.

[4] Andrei D Linde. Chaotic inflation, 1983.

[5] Andrei Linde. Quantum cosmology and the structure of inflationary universe. arXiv preprint gr-qc/9508019, 1995.

[6] Jerome Martin, Christophe Ringeval, and Vincent Vennin. Encyclopædia inflationaris. Physics of the Dark Universe, 5:75-235, 2014.

[7] David Wands. Multiple field inflation. In Inflationary cosmology, pages 275-304. Springer, 2008.
[8] Arjun Berera. Warm inflation. Physical Review Letters, 75(18):3218, 1995.

[9] Abhay Ashtekar and David Sloan. Loop quantum cosmology and slow roll inflation. Physics Letters B, 694(2):108-112, 2010.

[10] David Langlois and Sebastien Renaux-Petel. Perturbations in generalized multi-field inflation. Journal of Cosmology and Astroparticle Physics, 2008(04):017, 2008.

[11] Alexey Golovnev, Viatcheslav Mukhanov, and Vitaly Vanchurin. Vector inflation. Journal of Cosmology and Astroparticle Physics, 2008(06):009, 2008.

[12] MR Setare and V Kamali. Warm vector inflation. Physics Letters B, 726(1-3):56-65, 2013.

[13] Panagiota Kanti, Radouane Gannouji, and Naresh Dadhich. Gauss-bonnet inflation. Physical Review D, 92(4):041302, 2015.

[14] Gia Dvali and S-H Henry Tye. Brane inflation. Physics Letters B, 450(1-3):72-82, 1999. 
[15] Stephon Alexander, Antonino Marciano, and David Spergel. Chern-simons inflation and baryogenesis. Journal of Cosmology and Astroparticle Physics, 2013(04):046, 2013.

[16] N Aghanim, Yashar Akrami, M Ashdown, J Aumont, C Baccigalupi, M Ballardini, AJ Banday, RB Barreiro, N Bartolo, S Basak, et al. Planck 2018 results. vi. cosmological parameters. arXiv preprint arXiv:180\%.06209, 2018.

[17] Yashar Akrami, Frederico Arroja, M Ashdown, J Aumont, C Baccigalupi, M Ballardini, AJ Banday, RB Barreiro, N Bartolo, S Basak, et al. Planck 2018 resultsx. constraints on inflation. Astronomy $\& 5$ Astrophysics, 641:A10, 2020.

[18] Y Akrami, F Arroja, M Ashdown, J Aumont, C Baccigalupi, M Ballardini, AJ Banday, RB Barreiro, N Bartolo, S Basak, et al. Planck 2018 results. i. overview and the cosmological legacy of planck. arXiv preprint arXiv:180\%.06205, 2018.

[19] Nabila Aghanim, Y Akrami, F Arroja, M Ashdown, J Aumont, C Baccigalupi, M Ballardini, AJ Banday, RB Barreiro, N Bartolo, et al. Planck 2018 results. Astronomy and Astrophysics-AESA, 641:A1, 2020.

[20] Jérôme Martin, Christophe Ringeval, Roberto Trotta, and Vincent Vennin. The best inflationary models after planck. Journal of Cosmology and Astroparticle Physics, 2014(03):039, 2014.

[21] PJE Peebles and A Vilenkin. Quintessential inflation. Physical Review D, 59(6):063505, 1999.

[22] Robert R Caldwell, Rahul Dave, and Paul J Steinhardt. Cosmological imprint of an energy component with general equation of state. Physical Review Letters, 80(8):1582, 1998.

[23] Shinji Tsujikawa. Quintessence: a review. Classical and Quantum Gravity, 30(21):214003, 2013.

[24] Ivaylo Zlatev, Limin Wang, and Paul J Steinhardt. Quintessence, cosmic coincidence, and the cosmological constant. Physical Review Letters, 82(5):896, 1999.

[25] MC Bento, Orfeu Bertolami, and Anjan A Sen. Generalized chaplygin gas, accelerated expansion, and dark-energy-matter unification. Physical Review D, 66(4):043507, 2002.

[26] Konstantinos Dimopoulos and Leonora DonaldsonWood. Warm quintessential inflation. Physics Letters B, 796:26-31, 2019.

[27] David Benisty and Eduardo I Guendelman. Quintessential inflation from lorentzian slow roll. arXiv preprint arXiv:2006.04129, 2020.

[28] Sourish Dutta and Robert J Scherrer. Hilltop quintessence. Physical Review D, 78(12):123525, 2008.

[29] Takeshi Chiba. Slow-roll thawing quintessence. Physical Review D, 79(8):083517, 2009.

[30] Carl L Gardner. Braneworld quintessential inflation and sum of exponentials potentials. arXiv preprint hepph/0701036, 2007.

[31] M Sami and Varun Sahni. Quintessential inflation on the brane and the relic gravity wave background. Physical Review D, 70(8):083513, 2004.

[32] Tomi Koivisto and David F Mota. Gauss-bonnet quintessence: Background evolution, large scale structure, and cosmological constraints. Physical Review D, 75(2):023518, 2007.

[33] Francesca Perrotta, Carlo Baccigalupi, and Sabino Matarrese. Extended quintessence. Physical Review D,
61(2):023507, 1999.

[34] Carsten van de Bruck, Konstantinos Dimopoulos, Chris Longden, and Charlotte Owen. Gauss-bonnetcoupled quintessential inflation. arXiv preprint arXiv:1707.06839, 2017.

[35] David Benisty, Eduardo I Guendelman, Emil Nissimov, and Svetlana Pacheva. Quintessential inflation with dynamical higgs generation as an affine gravity. Symmetry, 12(5):734, 2020.

[36] Michael B Green and John H Schwarz. Supersymmetrical dual string theory. Nuclear Physics B, 181(3):502530, 1981.

[37] Michael B Green and John H Schwarz. Supersymmetric dual string theory:(iii). loops and renormalization. Nuclear Physics B, 198(3):441-460, 1982.

[38] Michael B Green and John H Schwarz. Supersymmetric dual string theory:(ii). vertices and trees. Nuclear Physics B, 198(2):252-268, 1982.

[39] Steven S Gubser, Igor R Klebanov, and Alexander M Polyakov. Gauge theory correlators from non-critical string theory. Physics Letters B, 428(1-2):105-114, 1998.

[40] Nathan Seiberg and Edward Witten. String theory and noncommutative geometry. Journal of High Energy Physics, 1999(09):032, 1999.

[41] David Garfinkle, Gary T Horowitz, and Andrew Strominger. Charged black holes in string theory. Physical Review D, 43(10):3140, 1991.

[42] Leonard Susskind. The anthropic landscape of string theory. Universe or multiverse, pages 247-266, 2003.

[43] Joseph Polchinski. String theory: Volume 2, superstring theory and beyond. Cambridge university press, 1998.

[44] Dieter Lüst and Stefan Theisen. Lectures on string theory, volume 346. Springer, 1989.

[45] Liam McAllister and Eva Silverstein. String cosmology: a review. General Relativity and Gravitation, 40(23):565-605, 2008.

[46] M Gasperini and Gabriele Veneziano. Dilaton production in string cosmology. Physical Review D, 50(4):2519, 1994.

[47] Maurizio Gasperini and Gabriele Veneziano. The prebig bang scenario in string cosmology. Physics Reports, 373(1-2):1-212, 2003.

[48] Maurizio Gasperini. Elements of string cosmology, volume 36. Cambridge University Press Cambridge, 2007.

[49] Arkady A Tseytlin and C Vafa. Elements of string cosmology. Nuclear Physics B, 372(1-2):443-466, 1992.

[50] KD Krori, T Chaudhury, Chandra Rekha Mahanta, and Ajanta Mazumdar. Some exact solutions in string cosmology. General Relativity and Gravitation, 22(2):123130, 1990.

[51] Jonathan J Heckman, Craig Lawrie, Ling Lin, and Gianluca Zoccarato. F-theory and dark energy. Fortschritte der Physik, 67(10):1900057, 2019.

[52] Jonathan J Heckman, Craig Lawrie, Ling Lin, Jeremy Sakstein, and Gianluca Zoccarato. Pixelated dark energy. Fortschritte der Physik, 67(11):1900071, 2019.

[53] Cumrun Vafa. The string landscape and the swampland. arXiv preprint hep-th/0509212, 2005.

[54] Georges Obied, Hirosi Ooguri, Lev Spodyneiko, and Cumrun Vafa. de sitter space and the swampland. arXiv preprint arXiv:1806.08362, 2018.

[55] Hirosi Ooguri and Cumrun Vafa. Non-supersymmetric ads and the swampland. arXiv preprint 
arXiv:1610.01533, 2016.

[56] Jacob McNamara and Cumrun Vafa. Cobordism classes and the swampland. arXiv preprint arXiv:1909.10355, 2019.

[57] Alek Bedroya and Cumrun Vafa. Trans-planckian censorship and the swampland. arXiv preprint arXiv:1909.11063, 2019.

[58] Sumit K Garg and Chethan Krishnan. Bounds on slow roll and the de sitter swampland. Journal of High Energy Physics, 2019(11):75, 2019.

[59] Keshav Dasgupta, Maxim Emelin, Mir Mehdi Faruk, and Radu Tatar. De sitter vacua in the string landscape. arXiv preprint arXiv:1908.05288, 2019.

[60] Ulf H Danielsson and Thomas Van Riet. What if string theory has no de sitter vacua? International Journal of Modern Physics D, 27(12):1830007, 2018.

[61] Shamit Kachru, Renata Kallosh, Andrei Linde, and Sandip P Trivedi. De sitter vacua in string theory. Physical Review D, 68(4):046005, 2003.

[62] Hirosi Ooguri, Eran Palti, Gary Shiu, and Cumrun Vafa. Distance and de sitter conjectures on the swampland. Physics Letters B, 788:180-184, 2019.

[63] William H Kinney, Sunny Vagnozzi, and Luca Visinelli. The zoo plot meets the swampland: mutual (in) consistency of single-field inflation, string conjectures, and cosmological data. Classical and quantum gravity, 36(11):117001, 2019.

[64] Oem Trivedi. Swampland conjectures and single field inflation in modified cosmological scenarios. arXiv preprint arXiv:2008.05474, 2020.

[65] Hao Geng. A potential mechanism for inflation from swampland conjectures. Physics Letters B, page 135430, 2020.

[66] Marco Scalisi and Irene Valenzuela. Swampland distance conjecture, inflation and $\alpha$-attractors. Journal of High Energy Physics, 2019(8):160, 2019.

[67] Marco Scalisi. Inflation, higher spins and the swampland. Physics Letters B, 808:135683, 2020.

[68] Amjad Ashoorioon. Rescuing single field inflation from the swampland. Physics Letters B, 790:568-573, 2019.

[69] Alek Bedroya, Robert Brandenberger, Marilena Loverde, and Cumrun Vafa. Trans-planckian censorship and inflationary cosmology. Physical Review D, 101(10):103502, 2020.

[70] Suddhasattwa Brahma. Trans-planckian censorship, inflation, and excited initial states for perturbations. Physical Review D, 101(2):023526, 2020.

[71] Robert Brandenberger and Edward Wilson-Ewing. Strengthening the tcc bound on inflationary cosmology. Journal of Cosmology and Astroparticle Physics, 2020(03):047, 2020.

[72] Mansi Dhuria and Gaurav Goswami. Trans-planckian censorship conjecture and nonthermal post-inflationary history. Physical Review D, 100(12):123518, 2019.

[73] Vahid Kamali and Robert Brandenberger. Relaxing the tcc bound on inflationary cosmology? European Physical Journal C, 80(4):1-6, 2020.

[74] Heliudson Bernardo. Trans-planckian censorship conjecture in holographic cosmology. Physical Review D, 101(6):066002, 2020.

[75] Shuntaro Mizuno, Shinji Mukohyama, Shi Pi, Yun-Long Zhang, et al. Universal upper bound on the inflationary energy scale from the trans-planckian censorship conjecture. Physical Review D, 102(2):021301, 2020.
[76] Hao-Hao Li, Gen Ye, Yong Cai, and Yun-Song Piao. Trans-planckian censorship of multistage inflation and dark energy. Physical Review D, 101(6):063527, 2020.

[77] Wei-Chen Lin and William H Kinney. Transplanckian censorship and k-inflation. Physical Review D, 101(12):123534, 2020.

[78] Kai Schmitz. Trans-planckian censorship and inflation in grand unified theories. Physics Letters B, 803:135317, 2020.

[79] Arjun Berera, Suddhasattwa Brahma, and Jaime R Calderón. Role of trans-planckian modes in cosmology. arXiv preprint arXiv:2003.07184, 2020.

[80] Suddhasattwa Brahma. Trans-planckian censorship conjecture from the swampland distance conjecture. Physical Review D, 101(4):046013, 2020.

[81] Hiroki Matsui and Fuminobu Takahashi. Eternal inflation and swampland conjectures. Physical Review D, 99(2):023533, 2019.

[82] Konstantinos Dimopoulos. Steep eternal inflation and the swampland. Physical Review D, 98(12):123516, 2018.

[83] William H Kinney. Eternal inflation and the refined swampland conjecture. Physical review letters, 122(8):081302, 2019.

[84] Suddhasattwa Brahma and Sarah Shandera. Stochastic eternal inflation is in the swampland. arXiv preprint arXiv:1904.10979, 2019.

[85] Ziwei Wang, Robert Brandenberger, and Lavinia Heisenberg. Eternal inflation, entropy bounds and the swampland. arXiv preprint arXiv:190\%.08943, 2019.

[86] Oem Trivedi. Rejuvenating the hope of a swampland consistent inflated multiverse with tachyonic inflation in the high energy rs-ii braneworld. arXiv preprint arXiv:2101.00638, 2021.

[87] Arjun Berera and Jaime R Calderón. Trans-planckian censorship and other swampland bothers addressed in warm inflation. Physical Review D, 100(12):123530, 2019.

[88] Meysam Motaharfar, Vahid Kamali, and Rudnei O Ramos. Warm inflation as a way out of the swampland. Physical Review D, 99(6):063513, 2019.

[89] Suratna Das. Warm inflation in the light of swampland criteria. Physical Review D, 99(6):063514, 2019.

[90] Suratna Das, Gaurav Goswami, and Chethan Krishnan. Swampland, axions, and minimal warm inflation. Physical Review D, 101(10):103529, 2020.

[91] Suratna Das. Note on single-field inflation and the swampland criteria. Physical Review D, 99(8):083510, 2019.

[92] Suratna Das. Distance, de sitter and trans-planckian censorship conjectures: the status quo of warm inflation. Physics of the Dark Universe, 27:100432, 2020.

[93] Meysam Motaharfar, Vahid Kamali, and Rudnei O Ramos. Warm way out of the swampland. arXiv preprint arXiv:1810.02816.

[94] Abolhassan Mohammadi, Tayeb Golanbari, Haidar Sheikhahmadi, Kosar Sayar, Lila Akhtari, MA Rasheed, and Khaled Saaidi. Warm tachyon inflation and swampland criteria. Chinese Physics C, 44(9):095101, 2020.

[95] Lisa Randall and Raman Sundrum. An alternative to compactification. Physical Review Letters, 83(23):4690, 1999.

[96] Lisa Randall and Raman Sundrum. Large mass hierarchy from a small extra dimension. Physical review 
letters, 83(17):3370, 1999.

[97] Merab Gogberashvili. Hierarchy problem in the shelluniverse model. International Journal of Modern Physics D, 11(10):1635-1638, 2002.

[98] Baojiu Li, John D Barrow, and David F Mota. Cosmology of modified gauss-bonnet gravity. Physical Review D, 76(4):044027, 2007.

[99] Chia-Min Lin, Kin-Wang Ng, and Kingman Cheung. Chaotic inflation on the brane and the swampland criteria. Physical Review D, 100(2):023545, 2019.

[100] Vahid Kamali, Meysam Motaharfar, and Rudnei O Ramos. Warm brane inflation with an exponential potential: a consistent realization away from the swampland. Physical Review D, 101(2):023535, 2020.

[101] SD Odintsov and VK Oikonomou. Swampland implications of gw170817-compatible einstein-gauss-bonnet gravity. Physics Letters B, page 135437, 2020.

[102] Zhu Yi and Yungui Gong. Gauss-bonnet inflation and the string swampland. Universe, 5(9):200, 2019.

[103] Rathin Adhikari, Mayukh Raj Gangopadhyay, et al. Power law plateau inflation potential in the rs $i i$ braneworld evading swampland conjecture. arXiv preprint arXiv:2002.07061, 2020.

[104] Ralph Blumenhagen, Irene Valenzuela, and Florian Wolf. The swampland conjecture and f-term axion monodromy inflation. Journal of High Energy Physics, 2017(7):145, 2017.

[105] Abhay Ashtekar, Tomasz Pawlowski, and Parampreet Singh. Quantum nature of the big bang: improved dynamics. Physical Review D, 74(8):084003, 2006.

[106] Abhay Ashtekar, Tomasz Pawlowski, and Parampreet Singh. Quantum nature of the big bang: an analytical and numerical investigation. Physical Review D, 73(12):124038, 2006.

[107] F Gomez, P Minning, and P Salgado. Standard cosmology in chern-simons gravity. Physical Review D, 84(6):063506, 2011.

[108] Rafael Bravo, Gonzalo A Palma, and M Simón Riquelme. A tip for landscape riders: multi-field inflation can fulfill the swampland distance conjecture. Journal of Cosmology and Astroparticle Physics, 2020(02):004, 2020.

[109] Vikas Aragam, Sonia Paban, and Robert Rosati. Multifield inflation in high-slope potentials. Journal of Cosmology and Astroparticle Physics, 2020(04):022, 2020.

[110] Prateek Agrawal, Georges Obied, Paul J Steinhardt, and Cumrun Vafa. On the cosmological implications of the string swampland. Physics Letters B, 784:271-276, 2018.

[111] Michele Cicoli, Giuseppe Dibitetto, and Francisco G Pedro. Out of the swampland with multifield quintessence? Journal of High Energy Physics, 2020(10):1-17, 2020.

[112] Masahiro Ibe, Masahito Yamazaki, and Tsutomu T Yanagida. Quintessence axion revisited in light of swampland conjectures. Classical and Quantum Gravity, 36(23):235020, 2019.

[113] S David Storm and Robert J Scherrer. Swampland conjectures and slow-roll thawing quintessence. Physical Review D, 102(6):063519, 2020.

[114] MC David Marsh. The swampland, quintessence and the vacuum energy. Physics Letters B, 789:639-642, 2019.

[115] Yessenia Olguin-Trejo, Susha L Parameswaran, Gianmassimo Tasinato, and Ivonne Zavala. Runaway quintessence, out of the swampland. Journal of Cosmology and Astroparticle Physics, 2019(01):031, 2019.

[116] Lavinia Heisenberg, Matthias Bartelmann, Robert Brandenberger, and Alexandre Refregier. Dark energy in the swampland. Physical Review D, 98(12):123502, 2018.

[117] Carsten van de Bruck and Cameron C Thomas. Dark energy, the swampland, and the equivalence principle. Physical Review D, 100(2):023515, 2019.

[118] Maxim Emelin and Radu Tatar. Axion hilltops, kahler modulus quintessence and the swampland criteria. International Journal of Modern Physics A, 34(28):1950164, 2019.

[119] Suddhasattwa Brahma and Md Wali Hossain. Dark energy beyond quintessence: Constraints from the swampland. Journal of High Energy Physics, 2019(6):70, 2019.

[120] SD Odintsov and VK Oikonomou. Finite-time singularities in swampland-related dark-energy models. EPL (Europhysics Letters), 126(2):20002, 2019.

[121] Yashar Akrami, Renata Kallosh, Andrei Linde, and Valeri Vardanyan. The landscape, the swampland and the era of precision cosmology. Fortschritte der Physik, 67(1-2):1800075, 2019.

[122] Marco Raveri, Wayne Hu, and Savdeep Sethi. Swampland conjectures and late-time cosmology. Physical Review D, 99(8):083518, 2019.

[123] Yashar Akrami, Misao Sasaki, Adam R Solomon, and Valeri Vardanyan. Multi-field dark energy: cosmic acceleration on a steep potential. arXiv preprint arXiv:2008.13660, 2020.

[124] VK Oikonomou. Rescaled einstein-hilbert gravity from $f(r)$ gravity: Inflation, dark energy and the swampland criteria. arXiv preprint arXiv:2012.01312, 2020.

[125] Adam G. Riess et al. A 2.4\% Determination of the Local Value of the Hubble Constant. Astrophys. J., 826(1):56, 2016.

[126] Shadab Alam et al. The clustering of galaxies in the completed SDSS-III Baryon Oscillation Spectroscopic Survey: cosmological analysis of the DR12 galaxy sample. Mon. Not. Roy. Astron. Soc., 470(3):2617-2652, 2017.

[127] Edvard Mörtsell and Suhail Dhawan. Does the hubble constant tension call for new physics? Journal of Cosmology and Astroparticle Physics, 2018(09):025, 2018.

[128] Sunny Vagnozzi. New physics in light of the h 0 tension: An alternative view. Physical Review D, 102(2):023518, 2020.

[129] Eleonora Di Valentino, Alessandro Melchiorri, Olga Mena, and Sunny Vagnozzi. Interacting dark energy in the early 2020s: A promising solution to the h0 and cosmic shear tensions. Physics of the Dark Universe, 30:100666, 2020.

[130] Eleonora Di Valentino, Alessandro Melchiorri, Olga Mena, and Sunny Vagnozzi. Nonminimal dark sector physics and cosmological tensions. Physical Review D, 101(6):063502, 2020.

[131] Luca Visinelli, Sunny Vagnozzi, and Ulf Danielsson. Revisiting a negative cosmological constant from lowredshift data. Symmetry, 11(8):1035, 2019.

[132] Eoin Ó Colgáin and Hossein Yavartanoo. Testing the swampland: H0 tension. Physics Letters B, 797:134907, 2019 . 
[133] Aritra Banerjee, Haiying Cai, Lavinia Heisenberg, Eoin Ó Colgáin, MM Sheikh-Jabbari, and Tao Yang. Hubble sinks in the low-redshift swampland. arXiv preprint arXiv:2006.00244, 2020.

[134] Chien-I Chiang, Jacob M Leedom, and Hitoshi Murayama. What does inflation say about dark energy given the swampland conjectures? Physical Review D, 100(4):043505, 2019.

[135] Sergio del Campo. Approach to exact inflation in modified friedmann equation. Journal of Cosmology and Astroparticle Physics, 2012(12):005, 2012.

[136] Oem Trivedi. The exact solution approach to warm inflation. arXiv preprint arXiv:2008.05899, 2020.

[137] William H Kinney. Hamilton-jacobi approach to nonslow-roll inflation. Physical Review D, 56(4):2002, 1997.

[138] Dark Energy Survey Collaboration: Fermilab, University of Illinois at Urbana-Champaign, University of Chicago, Lawrence Berkeley National Laboratory, Cerro-Tololo Inter-American Observatory, and Brenna Flaugher. The dark energy survey. International Journal of Modern Physics A, 20(14):3121-3123, 2005.

[139] Brenna Flaugher and Chris Bebek. The dark energy spectroscopic instrument (desi). In Ground-based and Airborne Instrumentation for Astronomy $V$, volume 9147, page 91470S. International Society for Optics and Photonics, 2014.

[140] Masahiro Takada, Richard S Ellis, Masashi Chiba, Jenny E Greene, Hiroaki Aihara, Nobuo Arimoto, Kevin
Bundy, Judith Cohen, Olivier Doré, Genevieve Graves, et al. Extragalactic science, cosmology, and galactic archaeology with the subaru prime focus spectrograph. Publications of the Astronomical Society of Japan, 66(1), 2014.

[141] René J Laureijs, Ludovic Duvet, Isabel Escudero Sanz, Philippe Gondoin, David H Lumb, Tim Oosterbroek, and Gonzalo Saavedra Criado. The euclid mission. In Space Telescopes and Instrumentation 2010: Optical, Infrared, and Millimeter Wave, volume 7731, page 77311H. International Society for Optics and Photonics, 2010.

[142] Satoshi Miyazaki, Yutaka Komiyama, Hidehiko Nakaya, Yukiko Kamata, Yoshi Doi, Takashi Hamana, Hiroshi Karoji, Hisanori Furusawa, Satoshi Kawanomoto, Tomoki Morokuma, et al. Hyper suprime-cam. In Ground-based and Airborne Instrumentation for Astronomy $I V$, volume 8446, page 84460Z. International Society for Optics and Photonics, 2012.

[143] LSST Dark Energy Science Collaboration et al. Large synoptic survey telescope: dark energy science collaboration. arXiv preprint arXiv:1211.0310, 2012.

[144] D Spergel, N Gehrels, C Baltay, D Bennett, J Breckinridge, M Donahue, A Dressler, BS Gaudi, T Greene, O Guyon, et al. Wide-field infrarred survey telescopeastrophysics focused telescope assets wfirst-afta 2015 report. arXiv preprint arXiv:1503.03757, 2015. 